\title{
Quadratic separation for feedback connection of an uncertain matrix and an implicit linear transformation
}

\author{
Dimitri Peaucelle, Didier Henrion *and Denis Arzelier \\ LAAS-CNRS \\ 7 avenue du colonel Roche, 31077 Toulouse, FRANCE \\ Tel. 0561336309 fax: 0561336969 \\ email: \{ peaucelle, henrion, arzelier \}@laas.fr
}

23rd September 2004

\begin{abstract}
: Topological separation is investigated in the case of an uncertain time-invariant matrix interconnected with an implicit linear transformation. A quadratic separator independent of the uncertainty is shown to prove losslessly the closed-loop well-posedness. Several applications for descriptor systems are then given. First, some known results for stability and pole location are demonstrated in a new way. Second, contributions to robust stability analysis are exposed. All results are formulated as linear matrix inequalities (LMIs).
\end{abstract}

Keywords : quadratic separation, LMI, stability, descriptor system

*Also with the Faculty of Electrical Engineering, Czech Technical University in Prague, Technická 2, 16627 Prague 


\section{Introduction}

Well-posedness of feedback systems provides a fertile framework for stability analysis of non-linear and uncertain systems. An associated fundamental concept is topological separation [9]. It states that internal signals of a multivariable feedback connection of two systems $F$ and $G$ are unique and bounded under external disturbances if and only if the graph of $F$ is topologically separated from the inverse graph of $G$. While finding such topological separator is tricky in general, for several choices of systems $F$ and $G$ there exist, sometimes lossless [8], tractable techniques. Among these, major results for robust stability analysis are given in [4] and references therein.

In this paper we investigate a special case of feedback connections where the first system is a given implicit linear transformation,

$$
\mathcal{E} z=\mathcal{A} w
$$

and the second one is a complex valued uncertain matrix gain,

$$
w=\nabla z, \quad \nabla \in \mathbb{W} .
$$

No assumption is made on the set of possible uncertainties $\mathbb{W}$. The main result proves that well-posedness of such systems is losslessly assessed by a quadratic separator (the topological separator is a quadratic functional of $z$ and $w$ ) independent of the uncertainty $\nabla$. The result is a generalisation of corollary 1 in [4] to implicit linear transformations. Closely related results are lemma 4 in [5] and the full-block S-procedure theorem 1 in [10]. In fact they prove to be different and we choose to give a detailed proof of our result.

The remaining of the paper is dedicated to corollaries of the main result. Our goal is to illustrate how previously obtained stability analysis conditions for descriptor systems are related to topological separation. First we show that stability of $E \dot{x}=A x$ is equivalent to well-posedness of a feedback connection of $\nabla=s^{-1} \mathbb{1}, s^{-1} \in \mathbb{C}^{+}$with an implicit linear transformation. Quadratic separation with respect to this uncertainty set proves to be related to the existence of a quadratic Lyapunov certificate. Extensions to discretetime system stability as well as to pole location analysis are also givenand compared with $[7,3,2]$. 


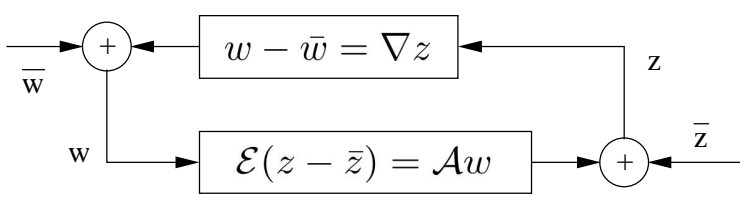

Figure 1: Feedback system

In the last section of the paper, corollaries for robust stability of descriptor systems are derived. More general than the framework by [6] and [12], the contribution considers rationally dependent models where uncertainties enter on both the $A$ and the $E$ matrices. To our knowledge these results are totally new. They allow to test stability of systems with structured uncertainty. More or less conservative tests are given that involve either constant or parameter-dependent Lyapunov functions. We believe that the contribution is original even when compared to similar results for usual LTI systems such as [5].

Notations: $\mathbb{R}^{m \times n}$ and $\mathbb{C}^{m \times n}$ are the sets of $m$-by- $n$ real and complex matrices respectively. $A^{T}$ is the transpose of the matrix $A$ and $A^{*}$ is its transpose conjugate. $A^{\perp}$ is a full rank matrix whose columns span the null-space of $A$. The columns of $A^{\circ}$ form an orthogonal basis of $A^{*}$, i.e. $\left[\begin{array}{ll}A^{\perp} & A^{\circ}\end{array}\right]$ is full rank. $\mathbb{1}$ and $\mathbb{O}$ are respectively the identity and the zero matrices of appropriate dimensions. For Hermitian matrices, $A>(\geq) B$ if and only if $A-B$ is positive (semi) definite.

\section{Main result}

Consider two possibly non-square matrices $\mathcal{E}$ and $\mathcal{A}$ and an uncertain matrix $\nabla$ with appropriate dimensions that belongs to some set $\mathbb{W}$. We make no assumption on the uncertainty set $\mathbb{W}$.

The feedback system in Figure 1 is said to be well-posed if for all uncertainties and all bounded input signals, the internal signals $w$ and $z$ are unique and bounded. Since only linear transformations enter the feedback 
system, $w$ and $z$ are necessarily unique if we can prove they are bounded. Well-posedness writes as

$$
\exists \bar{\gamma}>0: \begin{array}{r}
\forall(\bar{w}, \bar{z}) \\
\forall \nabla \in \mathbb{W}
\end{array},\left\|\left(\begin{array}{c}
w \\
z
\end{array}\right)\right\| \leq \bar{\gamma}\left\|\left(\begin{array}{c}
\bar{w} \\
\bar{z}
\end{array}\right)\right\| .
$$

Note that the equalities in Figure 1 imply

$$
(\mathcal{E}-\mathcal{A} \nabla) z=\mathcal{E} \bar{z}+\mathcal{A} \bar{w} .
$$

Well-posedness of the system states that for all admissible $\nabla \in \mathbb{W}$, the null space of $\mathcal{E}-\mathcal{A} \nabla$ is empty ( $\mathcal{E}-\mathcal{A} \nabla$ is non-singular if square).

Lemma 1 Well-posedness of the system in Figure 1 is equivalent to wellposedness of the feedback connected systems given by

$$
\left\{\begin{array}{l}
w-\hat{w}=\nabla \mathcal{E}^{\circ} y \\
\mathcal{E E}^{\circ}(y-\hat{y})-\mathcal{A} w=0
\end{array}\right.
$$

Proof: Write $z$ and $\bar{z}$ in the $\left[\begin{array}{cc}\mathcal{E}^{\perp} & \mathcal{E}^{\circ}\end{array}\right]$ basis, i.e. $z=\mathcal{E}^{\perp} y_{1}+\mathcal{E}^{\circ} y$, $\bar{z}=\mathcal{E}^{\perp} \hat{y}_{1}+\mathcal{E}^{\circ} \hat{y}$. Then take $\hat{w}=\bar{w}-\nabla \mathcal{E}^{\perp} y_{1}$. Equations of Figure 1 are equivalent to those in (2).

Thanks to this technical lemma, well-posedness of the considered system is equivalently written for a similar system where $\mathcal{E} \mathcal{E}^{\circ}$ is a, possibly non square, full rank matrix. Now the main result is stated.

Theorem 1 The uncertain feedback system of Figure 1 is well-posed if and only if there exists a Hermitian matrix $\Theta=\Theta^{*}$ satisfying both conditions

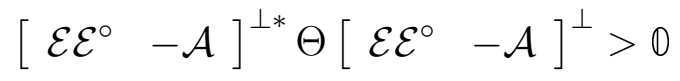

$$
\begin{aligned}
& {\left[\begin{array}{ll}
\mathbb{1} & \mathcal{E}^{\circ *} \nabla^{*}
\end{array}\right] \Theta\left[\begin{array}{c}
\mathbb{1} \\
\nabla \mathcal{E}^{\circ}
\end{array}\right] \leq \mathbb{0} \quad, \quad \forall \nabla \in \mathbb{W} .}
\end{aligned}
$$

If $\mathcal{E}$ and $\mathcal{A}$ are real matrices, the equivalence still holds with $\Theta$ restricted to be a real matrix. 
Proof of sufficiency: Assume (3) holds. It implies the existence of some positive scalar $\epsilon$ such that

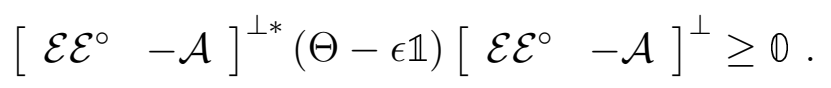

By definition of $\left[\mathcal{E E}^{\circ}-\mathcal{A}\right]^{\perp}$ and assuming (4) holds, one gets for all vectors that satisfy $(2)$

$$
\left\{\begin{array}{l}
\left(\begin{array}{c}
y-\hat{y} \\
w
\end{array}\right)^{*}(\Theta-\epsilon \mathbb{1})\left(\begin{array}{c}
y-\hat{y} \\
w
\end{array}\right) \geq 0 \\
\left(\begin{array}{c}
y \\
w-\hat{w}
\end{array}\right)^{*} \Theta\left(\begin{array}{c}
y \\
w-\hat{w}
\end{array}\right) \leq 0 .
\end{array}\right.
$$

Combining both inequalities, implies a quadratic constraint on the vector $X=\left(\begin{array}{ll|ll}w^{*} & y^{*} & \hat{w}^{*} & \hat{z}^{*}\end{array}\right)^{*}$ such as

$$
X^{*}\left[\begin{array}{cc}
\epsilon \mathbb{1} & T_{1} \\
T_{1}^{*} & T_{2}
\end{array}\right] X \leq \mathbb{0} .
$$

Take any $\tilde{\epsilon}$ such that $\epsilon>\tilde{\epsilon}>0$ and take a sufficiently large $\tilde{\gamma}>0$ such that

$$
\left[\begin{array}{cc}
\tilde{\epsilon} \mathbb{1} & \mathbb{0} \\
\mathbb{0} & -\tilde{\gamma} \mathbb{1}
\end{array}\right] \leq\left[\begin{array}{cc}
\epsilon \mathbb{1} & T_{1} \\
T_{1}^{*} & T_{2}
\end{array}\right]
$$

Well-posedness condition (1) is then proved for system (2) with $\bar{\gamma}=\tilde{\gamma} / \tilde{\epsilon}$. Lemma 1 concludes the proof of sufficiency.

Proof of necessity: Assume the system in Figure 1 is well-posed and equivalently that (2) is well-posed. First, note that if inequality (1) holds for $\bar{\gamma}$ it also holds for all $\gamma \geq \bar{\gamma}$. Well-posedness of (2) therefore implies that, for all $\gamma \geq \bar{\gamma}$ and for all $\nabla \in \mathbb{W}$, the equality constraint on $X$

$$
\overbrace{\left[\begin{array}{cc|cc}
-\mathbb{1} & \nabla \mathcal{E}^{\circ} & \mathbb{1} & \mathbb{0} \\
\mathcal{A} & -\mathcal{E E}^{\circ} & \mathbb{0} & \mathcal{E E}^{\circ}
\end{array}\right]}^{\mathcal{M}} X=0
$$

implies that the quadratic constraint

$$
X^{*}\left[\begin{array}{cc}
\mathbb{1} & 0 \\
0 & -\gamma \mathbb{1}
\end{array}\right] X \leq \mathbb{0}
$$


holds. Due to Finsler's lemma [11], it is equivalent to

$$
\mathcal{M}^{\perp *}\left[\begin{array}{cc}
\mathbb{1} & 0 \\
0 & -\gamma \mathbb{1}
\end{array}\right] \mathcal{M}^{\perp} \leq \mathbb{0} .
$$

Define $N_{1}$ and $N_{2}$ the partitions of $\left[\begin{array}{ll}\mathcal{E} & -\mathcal{A}\end{array}\right]^{\perp}$ such that

$$
\left[\begin{array}{ll}
\mathcal{E} \mathcal{E}^{\circ}-\mathcal{A}
\end{array}\right]^{\perp}=\left[\begin{array}{c}
N_{1} \\
N_{2}
\end{array}\right], \mathcal{E} \mathcal{E}^{\circ} N_{1}-\mathcal{A} N_{2}=\mathbb{O},
$$

$R=N_{1}^{*} N_{1}+N_{2}^{*} N_{2}$ and $Q=\gamma R-N_{2}^{*} N_{2}$. Since $\mathcal{E} \mathcal{E}^{\circ}$ is full rank, $N_{2}$ is also full rank. One can take

$$
\mathcal{M}^{\perp}=\left[\begin{array}{cc}
\mathbb{0} & N_{2} \\
\mathbb{1} & \mathbb{0} \\
\hline-\nabla \mathcal{E}^{\circ} & N_{2} \\
\mathbb{1} & -N_{1}
\end{array}\right]
$$

and the inequality (5) writes

$$
\left[\begin{array}{cc}
\mathbb{1}-\gamma \mathbb{1}-\gamma \mathcal{E}^{\circ *} \nabla^{*} \nabla \mathcal{E}^{\circ} & \gamma \mathcal{E}^{\circ *} \nabla^{*} N_{2}+\gamma N_{1} \\
\gamma N_{2}^{*} \nabla \mathcal{E}^{\circ}+\gamma N_{1}^{*} & -Q
\end{array}\right] \leq \mathbb{0} .
$$

Take $\gamma$ sufficiently large such that $Q>\mathbb{O}$. Applying a Schur complement argument on the block $-Q$, one gets inequality (4) where

$$
\Theta=\left[\begin{array}{cc}
\mathbb{1}-\gamma \mathbb{1}+\gamma^{2} N_{1} Q^{-1} N_{1}^{*} & \gamma^{2} N_{1} Q^{-1} N_{2}^{*} \\
\gamma^{2} N_{2} Q^{-1} N_{1}^{*} & -\gamma \mathbb{1}+\gamma^{2} N_{2} Q^{-1} N_{2}^{*}
\end{array}\right] .
$$

This matrix is real if $\mathcal{E}$ and $\mathcal{A}$ are real matrices. Let us prove now that (3) also holds.

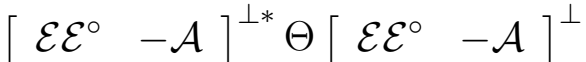

$$
\begin{aligned}
& =N_{1}^{*} N_{1}-\gamma R+\gamma^{2} R\left(\gamma R-N_{2}^{*} N_{2}\right)^{-1} R
\end{aligned}
$$

Apply a first time the matrix inversion lemma to $\left(\gamma R-N_{2}^{*} N_{2}\right)^{-1}$ to get

$$
=N_{1}^{*} N_{1}+N_{2}^{*}\left(\mathbb{1}-N_{2}(\gamma R)^{-1} N_{2}^{*}\right)^{-1} N_{2}
$$

and a second time to $\left(\mathbb{1}-N_{2}(\gamma R)^{-1} N_{2}^{*}\right)^{-1}$ to conclude

$$
\begin{aligned}
& =N_{1}^{*} N_{1}+N_{2}^{*}\left(\mathbb{1}+N_{2}\left(\gamma R-N_{2}^{*} N_{2}\right)^{-1} N_{2}^{*}\right) N_{2} \\
& =R+N_{2}^{*} N_{2} Q^{-1} N_{2}^{*} N_{2}>\mathbb{0} .
\end{aligned}
$$

Both inequalities (3) and (4) hold for any $\gamma$ sufficiently large to ensure $Q>\mathbb{0}$. 


\section{$3 \quad$ Stability of descriptor systems}

\subsection{Continuous-time descriptor systems}

A linear descriptor system characterised by the state-space equation

$$
E \dot{x}=A x
$$

fits the feedback system framework of Figure 1 if one considers

$$
\begin{aligned}
z=\dot{x}, \quad w=x, & \mathcal{E}=E, \quad \mathcal{A}=A \\
\nabla=s^{-1} \mathbb{1}, & s^{-1} \in \mathbb{C}^{+} .
\end{aligned}
$$

A quadratic separator for this set of uncertainties can be chosen as

$$
\Theta=\left[\begin{array}{cc}
0 & -E^{\circ T} P \\
-P E^{\circ} & \mathbb{O}
\end{array}\right] \quad, \quad E^{\circ T} P E^{\circ}>\mathbb{0} .
$$

Based on results of [8], the choice of such a structure for the separator may be proved to be non conservative.

Corollary 1 The descriptor system (6) is admissible, i.e. regular, stable and impulse free [7], if and only if the following LMI conditions hold

$$
\begin{aligned}
& E^{\circ T} P E^{\circ}>\mathbb{0} \\
& {\left[\begin{array}{cc}
E E^{\circ} & -A
\end{array}\right]^{\perp T}\left[\begin{array}{cc}
0 & E^{\circ T} P \\
P E^{\circ} & \mathbb{O}
\end{array}\right]\left[\begin{array}{cc}
E E^{\circ} & -A
\end{array}\right]^{\perp}<\mathbb{0} \text {. }}
\end{aligned}
$$

Proof of necessity: One way to prove necessity of corollary 1 is to show how this result is related to the LMI conditions of [7]

$$
E^{T} X^{T}=X E \geq \mathbb{0}, A^{T} X^{T}+X A<\mathbb{0} .
$$

For a stable descriptor system there exist two non singular matrices $V$ and $U$ such that

$$
V E U=\left[\begin{array}{ll}
\mathbb{1} & 0 \\
0 & 0
\end{array}\right] \quad, \quad V A U=\left[\begin{array}{cc}
A_{1} & \mathbb{0} \\
0 & \mathbb{1}
\end{array}\right]
$$


Based on this factorisation take

$$
E^{\circ}=U\left[\begin{array}{l}
\mathbb{1} \\
\mathbb{0}
\end{array}\right] \quad, \quad\left[\begin{array}{ll}
E E^{\circ} & -A
\end{array}\right]^{\perp}=\left[\begin{array}{l}
A_{1} \\
E^{\circ}
\end{array}\right] .
$$

Replace these in (7) and pre and post-multiply the formula by $E^{\circ T}$ and $E^{\circ}$ respectively. The result is exactly the conditions of corollary 1 with $P=U^{-T} V^{-T} X^{T}$

Remark that conditions of corollary 1 imply to solve a smaller LMI problem in terms of number of variables and size of the constraints. This can prove more efficient for large scale problems. On the other hand, the constraints (7) may be useful when dealing with design problems [7], it is not yet the case for the new proposed result.

Remark also that the matrix $P$ in corollary 1 defines a quadratic Lyapunov function $V(x)=x^{T} P x$ such that $V(x)>0$ for all $x$ in the image of $E^{\circ}$, while $\dot{V}(x)<0$ for all $(\dot{x}, x)$ in the null space of $[E-A]$.

\subsection{Discrete-time descriptor systems and pole location}

The discrete-time case is very much similar to the continuous-time case. The state-space representation $E x_{k+1}=A x_{k}$ is identically modelled as a feedback system in Figure 1. The unique difference is the uncertainty set

$$
\nabla=s^{-1} \mathbb{1} \quad, \quad\left|s^{-1}\right| \leq 1
$$

Non-conservative separators may be parameterised as

$$
\Theta=\left[\begin{array}{cc}
-E^{\circ T} P E^{\circ} & 0 \\
0 & P
\end{array}\right] \quad, \quad E^{\circ T} P E^{\circ}>\mathbb{0} .
$$

Applying theorem 1, condition (3) with this choice of separator is a necessary and sufficient LMI condition for the stability of the discrete-time descriptor system. The result is related to the generalised discrete Lya-

punov inequality of [3] that writes $E^{T} X E>A^{T} X A, E^{T} X E \geq \mathbb{0}$, by taking $P=U^{-T} V^{-T} X V^{-1} U^{-1}$. 
The procedure can be extended to pole location analysis. For example, take regions of the complex plane described by a scalar quadratic inequality:

$$
\mathcal{D}=\left\{s \in \mathbb{C}: d_{1}+d_{2} s+d_{2}^{*} s^{*}+d_{3} s s^{*} \leq 0\right\} .
$$

Such regions are half-planes, interior of disks or exteriors of disks. The poles of $E \dot{x}=A x$ (i.e. values such that $\operatorname{rank}(E s-A)$ drops from its normal value) lie in $\mathcal{D}$ if the feedback system of Figure 1 is well-posed for all $s$ outside the region. Pole location analysis amounts to testing well-posedness with respect to the following uncertainty set:

$$
\mathbb{W}=\left\{s^{-1} \mathbb{1}: \begin{array}{l}
d_{1} s^{-1} s^{-*} \\
+d_{2} s^{-*} \\
+d_{2}^{*} s^{-1}+d_{3} \geq 0
\end{array}\right\} .
$$

Necessary and sufficient LMI condition for pole location analysis are then obtained applying theorem 1 with the following separator:

$$
\Theta=\left[\begin{array}{cc}
d_{3} E^{\circ T} P E^{\circ} & d_{2}^{*} E^{\circ T} P \\
d_{2} P E^{\circ} & d_{1} P
\end{array}\right], \quad E^{\circ T} P E^{\circ}>\mathbb{O} .
$$

For many other regions (as well as for unions of regions) separators can be chosen following the methodology in [2]. For intersections of regions, the procedure consists in proving pole location in each region independently.

\subsection{Polynomial systems}

If you consider a matrix differential equation of degree $d$ defined by

$$
\sum_{i=0}^{d} A_{i} x^{(i)}=0 \quad, \quad x \in \mathbb{R}^{n},
$$


stability is then equivalent to well-posedness of the system in Figure 1 with

$$
\begin{aligned}
& \mathcal{E}=\left[\begin{array}{cccc}
A_{d} & \mathbb{0} & \cdots & \mathbb{0} \\
\mathbb{0} & -\mathbb{1} & & \mathbb{0} \\
\vdots & & \ddots & \\
\mathbb{0} & \mathbb{0} & & -\mathbb{1}
\end{array}\right] \quad \nabla=s^{-1} \mathbb{1}_{d n} \\
& \mathcal{A}=-\left[\begin{array}{cccc}
A_{d-1} & \cdots & A_{1} & A_{0} \\
\mathbb{1} & & \mathbb{0} & \mathbb{0} \\
& \ddots & & \vdots \\
\mathbb{0} & & \mathbb{1} & \mathbb{0}
\end{array}\right] \quad s^{-1} \in \mathbb{C}^{+}
\end{aligned}
$$

In the case $A_{d}$ is full rank, stability and pole location LMI conditions obtained when applying theorem 1 to this system are exactly the same as the one proposed in $[2]$.

\section{Robust stability of descriptor systems}

Consider the following uncertain descriptor system

$$
\begin{aligned}
& \left(E_{1}+B \Delta\left(E_{3}-D \Delta\right)^{-1} E_{2}\right) \dot{x} \\
& \quad=\left(A+B \Delta\left(E_{3}-D \Delta\right)^{-1} C\right) x
\end{aligned}
$$

where the state-space model matrices are rational functions of the uncertain parameters $\Delta$ that are assumed to belong to a set $\Delta$. This model matches the framework of Figure 1 if one considers

$$
\overbrace{\left[\begin{array}{cc}
E_{1} & 0 \\
E_{2} & E_{3}
\end{array}\right]}^{\mathcal{E}}\left(\begin{array}{c}
\dot{x} \\
z_{\Delta}
\end{array}\right)=\overbrace{\left[\begin{array}{ll}
A & B \\
C & D
\end{array}\right]}^{\mathcal{A}}\left(\begin{array}{c}
x \\
w_{\Delta}
\end{array}\right)
$$

along with the set:

$$
\mathbb{W}=\left\{\left[\begin{array}{cc}
s^{-1} \mathbb{1}_{n} & \mathbb{0} \\
\mathbb{0} & \Delta
\end{array}\right]: s^{-1} \in \mathbb{C}^{+}, \Delta \in \mathbb{\Delta}\right\}
$$




\subsection{Unstructured uncertainty}

Assume the uncertainties are non-structured norm-bounded: $\Delta^{T} \Delta \leq \mathbb{1}$. A non conservative choice of quadratic separators for $\mathbb{W}$ is described by

$$
\begin{gathered}
\mathcal{E}_{1}^{\circ T} P \mathcal{E}_{1}^{\circ}>\mathbb{0}, \tau>0 \\
\Theta=\left[\begin{array}{cc|cc}
0 & \mathbb{0} & -\mathcal{E}_{1}^{\circ T} P & \mathbb{0} \\
\mathbb{0} & -\tau \mathcal{E}_{2}^{\circ T} \mathcal{E}_{2}^{\circ} & \mathbb{0} & \mathbb{0} \\
\hline-P \mathcal{E}_{1}^{\circ} & \mathbb{0} & \mathbb{0} & \mathbb{0} \\
\mathbb{0} & \mathbb{0} & \mathbb{0} & \tau \mathbb{1}
\end{array}\right]
\end{gathered}
$$

where $\mathcal{E}_{1}^{\circ}$ and $\mathcal{E}_{2}^{\circ}$ are the relevant row partitions of $\mathcal{E}$. Applying theorem 1 with this choice of separator, we get a necessary and sufficient LMI condition for robust stability of the uncertain descriptor system.

\subsection{Structured uncertainty}

Consider now the structured uncertainty such that $\Delta=\delta \mathbb{1}_{m}$ with $\delta$ real and norm-bounded, $|\delta| \leq 1$. Parametric uncertainty is assumed: $\delta$ is an unknown constant scalar. Based on mixed P-separators and vertex-separators [4], define the following structured separator

$$
\Theta=\left[\begin{array}{cc|cc}
\mathbb{0} & \mathbb{0} & -\mathcal{E}_{1}^{\circ T} P & \mathbb{0} \\
\mathbb{0} & \mathcal{E}_{2}^{\circ T} \Theta_{1} \mathcal{E}_{2}^{\circ} & \mathbb{0} & \mathcal{E}_{2}^{\circ T} \Theta_{2} \\
\hline-P \mathcal{E}_{1}^{\circ} & \mathbb{0} & \mathbb{0} & \mathbb{0} \\
\mathbb{0} & \Theta_{2}^{T} \mathcal{E}_{2}^{\circ} & \mathbb{0} & \Theta_{3}
\end{array}\right]
$$

constrained by the LMIs

$$
\begin{aligned}
& \mathcal{E}_{1}^{\circ T} P \mathcal{E}_{1}^{\circ}>\mathbb{0}, \quad, \quad \mathcal{E}_{2}^{\circ T} \Theta_{3} \mathcal{E}_{2}^{\circ} \geq \mathbb{0} \\
& \mathcal{E}_{2}^{\circ T}\left(\Theta_{1}+\Theta_{2}+\Theta_{2}^{T}+\Theta_{3}\right) \mathcal{E}_{2}^{\circ} \leq \mathbb{0} \\
& \mathcal{E}_{2}^{\circ T}\left(\Theta_{1}-\Theta_{2}-\Theta_{2}^{T}+\Theta_{3}\right) \mathcal{E}_{2}^{\circ} \leq \mathbb{0}
\end{aligned}
$$

Corollary 2 For $\mathcal{E}$ and $\mathcal{A}$ defined in (9), if there exist a matrix $P \in \mathbb{R}^{n}$ and matrices $\Theta_{i=1,2,3} \in \mathbb{R}^{m}$ that satisfy (3), (10) and (11), then the uncertain system (8) is stable for all $\Delta=\delta \mathbb{1},|\delta| \leq 1$. 
Now notice that the system equivalently writes as

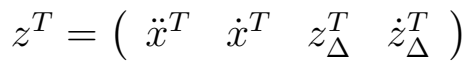

$$
\begin{aligned}
& w^{T}=\left(\begin{array}{llll}
\dot{x}^{T} & x^{T} & w_{\Delta}^{T} & \dot{w}_{\Delta}^{T}
\end{array}\right) \\
& \overbrace{\left[\begin{array}{cccc}
\mathbb{0} & \mathbb{1} & \mathbb{0} & \mathbb{0} \\
\mathbb{0} & E_{1} & \mathbb{0} & \mathbb{0} \\
\mathbb{0} & E_{2} & E_{3} & \mathbb{0} \\
E_{1} & \mathbb{0} & \mathbb{0} & \mathbb{0} \\
E_{2} & \mathbb{0} & \mathbb{0} & E_{3}
\end{array}\right]}^{\mathcal{E}} z=\overbrace{\left[\begin{array}{cccc}
\mathbb{1} & \mathbb{0} & \mathbb{0} & 0 \\
0 & A & B & 0 \\
\mathbb{0} & C & D & 0 \\
A & \mathbb{0} & \mathbb{0} & B \\
C & 0 & 0 & D
\end{array}\right]}^{\mathcal{A}} w
\end{aligned}
$$

with a feedback operator

$$
\nabla=\left[\begin{array}{cc}
s^{-1} \mathbb{1}_{2 n} & \mathbb{0} \\
\mathbb{0} & \delta \mathbb{1}_{2 m}
\end{array}\right] .
$$

An other corollary to theorem 1 is:

Corollary 3 For $\mathcal{E}$ and $\mathcal{A}$ defined in (12), if there exist a matrix $P \in \mathbb{R}^{2 n}$ and matrices $\Theta_{i=1,2,3} \in \mathbb{R}^{2 m}$ that satisfy (3), (10) and (11), then the uncertain system (8) is stable for all $\Delta=\delta \mathbb{1},|\delta| \leq 1$.

Both corollaries 2 and 3 are conservative results for robust stability analysis with respect to structured uncertainty. It can be proved (taking relevant sub-blocks in the LMIs) that conditions of corollary 2 hold if those of corollary 3 hold. Hence corollary 3 is less conservative than corollary 2 at the expense of increased numerical complexity (about four times as many variables and twice as large LMIs).

In case of usual LTI systems $\left(E_{1}=\mathbb{1}, E_{2}=\mathbb{O}, E_{3}=\mathbb{1}\right)$, close results were obtained by [5]. For example, our corollary 3 is similar to their theorem 5. Both results read as analysis conditions where stability is proved with a parameter-dependent Lyapunov function $V_{\Delta}(x)=x^{T} P(\Delta) x$. But the choice of $P_{\Delta}$ is different. In corollary 3 stability of $\dot{x}=A(\Delta) x=(A+B \Delta(\mathbb{1}-$ $\left.D \Delta)^{-1} C\right) x$ is assessed by

$$
P_{\Delta}=\left[\begin{array}{c}
A(\Delta) \\
\mathbb{1}
\end{array}\right]^{T} P\left[\begin{array}{c}
A(\Delta) \\
\mathbb{1}
\end{array}\right]
$$


while in [5] the parameter-dependent Lyapunov matrix is such that

$$
P_{\Delta}=\left[\begin{array}{c}
\Delta(\mathbb{1}-D \Delta)^{-1} C \\
\mathbb{1}
\end{array}\right]^{T} P\left[\begin{array}{c}
\Delta(\mathbb{1}-D \Delta)^{-1} C \\
\mathbb{1}
\end{array}\right]
$$

\section{Conclusion}

A novel quadratic separation framework for feedback connected systems with implicit linear transformation is described. Directly related results are derived for descriptor systems both for robust stability and pole location analysis. Only two special cases of uncertainties were considered but extensions can be obtained for more complex and time-varying structured uncertainties following results of $[5,1]$. Prospective work will be dedicated to analysing the relative conservatism of several independent results when stability is proved with parameter-dependent Lyapunov certificates.

Acknowledgement: D. Henrion acknowledges support from project No. ME 698/2003 of the Ministry of Education of the Czech Republic.

\section{References}

[1] M. Dettori and C.W. Scherer. Robust stability analysis for parameter dependent systems using full block S-procedure. In Selected Topics in Identification, Modelling and Control, volume 11, pages 17-25. Delft University Press, December 1998.

[2] D. Henrion, O. Bachelier, and M. Šebek. D-stability of polynomial matrices. Int. J. Control, 74(8):845-856, May 2001.

[3] K.-L. Hsiung. On the discrete-time bounded real lemma for descriptor systems. In IEEE Conference on Decision and Control, pages 289-290, Tampa, Florida, December 1998. 
[4] T. Iwasaki and S. Hara. Well-posedness of feedback systems: Insights into exact robustness analysis and approximate computations. IEEE Trans. on Automat. Control, 43(5):619-630, 1998.

[5] T. Iwasaki and G. Ishibata. LPV system analysis via quadratic separator for uncertain implicit systems. IEEE Trans. on Automat. Control, 46(8):1195-1207, August 2001.

[6] C.-L. Lin. On the stability of uncertain linear descriptor systems. Journal of the Franklin Institute, 336:549-564, 1999.

[7] I. Masubuchi, Y. Kamitane, A. Ohara, and N. Suda. $H_{\infty}$ control for descriptor systems: A matrix inequalities approach. Automatica, 33(4):669-673, 1997.

[8] G. Meinsma, Y. Shrivastava, and M. Fu. A dual formulation of mixed $\mu$ and on the losslessness of $(D, G)$-scaling. IEEE Trans. on Automat. Control, 42(7):1032-1036, 1997.

[9] M.G. Safonov. Stability and Robustness of Multivariable Feedback Systems. Signal Processing, Optimization, and Control. MIT Press, 1980.

[10] C. Scherer. A full block S-procedure with applications. In IEEE Conference on Decision and Control, pages 2602-2607, San Diego, CA, December 1997.

[11] R.E. Skelton, T. Iwazaki, and K. Grigoriadis. A unified Approach to Linear Control Design. Taylor and Francis series in Systems and Control, 1998.

[12] S. Xu, J. Lam, and C. Yang. Robust $H_{\infty}$ control for uncertain discrete singular systems with pole placement in a disk. Systems $\&$ Control Letters, 43:85-93, 2001. 